\title{
Image Attributes and Diffusion via Twitter: The Case of \#guncontrol
}

\author{
Michael A. Stefanone, Ph.D. \& \\ Gregory D. Saxton, Ph.D. \\ University at Buffalo \\ ms297;gdsaxton@buffalo.edu
}

\author{
Michael J. Egnoto \\ Ithaca College \\ megnoto@ithaca.edu \\ Wayne X. Wei \\ University at Buffalo, \\ weiaixu@buffalo.edu
}

\author{
Yun Fu, Ph.D. \\ Northeastern University \\ yunfu@ece.neu.edu
}

\begin{abstract}
We report on a study exploring how Twitter user attributes and the characteristics of the images they share online influence the diffusion of those images. Two-hundred and ninety unique images were collected from Twitter in October 2013 associated with the guncontrol hashtag [\#guncontrol]. A coding protocol was developed and images classified based on frame (attribute, goal, or risk), appeal (e.g. fear, humor), and valence (positive or negative). Results indicate that shared images with attribute frames, fear and humor appeals, and positive valence are retweeted more often. Also retweeted more frequently are messages from users with larger networks and whose tweets contain hashtags. Results also show a significant negative relationship between the time since the last major shooting event in the United States and the likelihood that messages with images are retweeted. These results are discussed in the context of evolving mass media systems and online social networks.
\end{abstract}

\section{Introduction}

Despite the growing concentration and reach of traditional mass media conglomerates [28], individuals are spending more time online interacting with new and social media technologies to access information [34]. Online social media platforms like Twitter are changing how we create, broadcast and receive information about almost everything that interests us, and are displacing the time spent with traditional, mainstream information sources. While global traffic statistics for traditional information sources like nytimes.com continue to plummet (nytimes.com), the popularity and use of social media platforms is at an all time high (Alexa.com, 2014).

For example, Facebook users upload in excess of 300 million images (Facebook, 2013) and Twitter users exchange more than 500 million tweets daily (Twitter, 2013). Among those tweets, 36\% contain images. Image sharing is one of the most common behaviors online today, and research shows that content containing images drives increased user engagement, opposed to pure text $[4,38]$.

While there is an emergent scholarship on how text-based content drives diffusion [19], the impact image characteristics have on information diffusion remains largely unexplored. Thus, this study is designed to address this gap in the literature. We investigate images shared via Twitter using \#guncontrol. Gun control was chosen because of how active this topic is in the public sphere as is evident by a recent PEW report [53] titled Gun Control and the Media. We draw on classic persuasion literature to develop a coding scheme for image characteristics and apply it to manually code all images along appeal, frame and valence attributes. We then investigate the relationship between these image attributes and diffusion (or, retweets) while controlling for Twitter user network characteristics and temporal factors.

\section{Mass Media}

Media effects are ubiquitous and nuanced, and theoretical models are constantly evolving to better describe the mechanisms by which media influence audiences [39]. At the core of mass media research is the media effect on individual attitude and behavior [40]. Mass media effects like framing focus on how highlighting certain aspects of news stories promote predictable interpretations of those stories by audiences. Other models like two-step flow suggest that information and opinions spread through social systems that are mediated by opinion leaders. The spread of information via two-step flow processes mirrors diffusion of innovations processes. The diffusion literature is well suited to investigating the complexities of media effects research [26, 39, 49]. 


\subsection{Diffusion}

Information diffusion across social media requires attention to message content (how messages are constructed) and the social context used to interpret the messages (topic salience, timeliness of messages, etc.) to be successful; additional user-level characteristics like activity frequency, network structure, and location are also important predictors of message diffusion [11]. Research shows that messages that entertain audiences, use images, and elicit emotions diffuse more effectively than other messages shared via social media $[46,48]$. Though these studies describe effective information diffusion, they shed little light on the process that these characteristics use to improve information flow.

Tools like Twitter enable users to broadcast content to large audiences (or, their followers), and consume content tailored to their individual interests and viewpoints. Social media tools are unique from other forms of computer-mediated communication in that they make associations between individuals in the form of friends or following behavior public [43]. Individuals have full control over social media content because they have the ability to approve of topics, sources, and types of content while ignoring less desirable information [37]. The result of user control on information consumption forms an egocentric view of the internet where users filter unwanted information [18]. As users have more choice and ability to be selective, and users are more selective in what content they pay attention to, it becomes imperative to examine what type of information garner audience attention.

We propose that to adequately address the evolution of text-based influence techniques to visual formats, scholars must build from foundations established in content analysis and persuasion. These elements include a range of different categories of appeals, framing characteristics, and image valence $[20,23,51]$.

Information diffusion focuses on the spread of ideas between people and throughout social systems [35]. Diffusion studies generally investigate the introduction and adoption of ideas, opinions, technologies, or products, collectively called innovations [35, 47]. Messages are information containers that create shared meaning between sources and receivers. Messages convey and elicit a variety of experiences, including valence. Valence refers to emotional positivity or negativity messages solicit from consumers.

Valence alone does not provide sufficient information to fully convey a message. Messages must have a purpose with structure and emphasis used to create meaning in recipients. The purpose of a message is the topic or goal. Structure and emphasis give form to topics and goals, and give messages influential capabilities. The structure messages take is analogous to appeals. Appeals are the format used for requests. Appeals can be rational or emotional in basis, appealing to either logic or emotive message processing [51]. Emphasis is similar to framing [21]. Framing highlights a component of the message. Appeals, frames, and valence are known to be effective tools in increasing information diffusion [25, 33, 42]. Therefore, it makes sense to conceptualize messages as being composed of appeals, frames, and valences, and to evaluate each of these characteristics to effectively communicate about a variety of topics.

Recall that the inclusion of images was related to increased information diffusion in the form of retweeting behavior on Twitter [4] as well as sharing behavior on Facebook [38]. However, scholarship linking specific image characteristics to diffusion via social media is lacking. General message construction characteristics including appeal, frame, and valence apply equally well to image coding. Hence, in this study we apply the appeal-framevalence framework to categorizing images shared via social media tools.

\subsection{Message Characteristics}

2.2.1. Appeals. Message receivers are engaged by appeals through emotion and reason. The nature of the appeal influences how messages are processed [41]. Appeals rely on users' past experience to help process information. These past experiences give message consumers cues on how they should react, internalize, and process the information presented to them. Cialdini [12] relates influence to past experience and expectations of reciprocity, commitment, scarcity, authority, social proof, and liking. All of these categories are tied closely to emotional and rational experiences. The purpose of those experiences is to evoke an emotional or rational response that contains a strong sense of feeling or logic. Therefore influence focuses on the ability to evoke previously established mental processing paths in message consumers. Several dominant appeals speak to the emotional and cognitive aspects of influence. Humor appeals rely on the emotional capacity for joyful affect with forms of humor and play being noted in social animals of all types [29, 32]. Fear appeals similarly rely on base emotional experiences.

The pervasiveness of fear and humor suggests deep cognitive connections to how certain 
components of messages are processed. Ramachandran and Blakeslee [32] note that fear and humor are linked expressions of emotion where an initial threat evokes a fearful response, but once the threat is discredited, joy in the form of laughter or humor is then expressed. Fear and humor appeals occupy a cognitive place where all humans can identify with them, and we may be evolutionarily biased to pay special attention to them. The power and universality of fear and humor likely means they have strong and accessible heuristics for cognitive processing. These pervasive routes are effective mechanisms to influence others. Thus the following hypotheses are proposed:

H1a: Images with fear appeals are positively related to retweeting frequency, and

H1b: Images with humor appeals are positively related to retweeting frequency, opposed to other appeals.

2.2.2. Framing. Framing is the process by which certain elements of messages are emphasized $[15,21]$ and can encourage a particular interpretation.[36] find that loss and gain frames are influential in driving public opinion on health-related issues and public perception of risk. Grabe and Bucy [16] find that the presentation of images in political formats is related to voting behavior. Framing is often used with different appeals (e.g., fear) to influence how information is evaluated [8].

Additional research by Krishnamurthy, Carter, and Blair [22] suggests that attribute frames are more effective than goal and risk frames in cases with positive valence. Attribute frames focus on a single component of a message. This often manifests as highlighting, either in text or in the photo, a particular point of emphasis the authors wish to draw attention to. Valence frames instead focus on the intended emotionality of a message, and may emphasize how people should feel (don't you feel bad?, would that make you feel good?). Finally, goal frames focus on outcomes and offer clear outcomes that readers should aspire towards (e.g., remove $\mathrm{X}$ for $\mathrm{Y}$ outcome). Taken together, this evidence suggests that images that incorporate attribute frames in particular should be positively associated with retweeting frequency. Thus,

$\mathrm{H} 2$ : Images with attribute frames are positively related to retweeting frequency, opposed to other frames.
2.2.3. Valence. Valence refers to whether message content solicits positive or negative feelings in message receivers [20]. Previous research by Maier [27] finds that message valence is a significant factor in how influence manifests in group communication such that increases in positive valence are related to adoption choices, regardless of quality. In terms of research on Twitter, Hansen, Arvidsson, Nielsen, and Colleoni [17] found that the most retweeted news content was associated with negative valence, while positive valence was more often associated with a range of non-news related content.

Bizer, Larsen, and Petty [6] find that negative frames enhance attitude strength in message receivers; however Lin and Pena [24] suggest that positive emotional messages are more popular than negative ones, and that emotional messages in general diffuse more widely opposed to directive messages. This evidence suggests that positive valence is an influential message characteristic. Thus,

H3: Images with positive message valence are positively related to retweeting frequency, opposed to negative valence.

Appeals, frames, and valence impact how information is attended to and internalized by individuals, and influence online sharing behavior. Message source characteristics also likely influence retweeting behavior as well. We review research on source characteristics below.

\subsection{Message Source Characteristics}

Careful consideration must also be given to the source of messages communicated via Twitter. Extant research shows that source characteristics like perceived expertise and trustworthiness are important predictors of attention given to news-related content [13]. In research on social media users are discussed with regards to identity, issue involvement, expertise, and position in social networks [52]. Users who are more connected to media and involved with technology are more successful at information diffusion [2, 52].

Scholars have explored various source attributes including trustworthiness, expertise and experience, carefulness, sincerity and neutrality [31]. In Twitter interactions, source attributes can be gauged by various indicators accessible from the profiles of Twitter users. For example, the number of followers is an indication of user connectivity [9] and a user's popularity and visibility [1], and is used for judging source competence [50]. In network terms, the 
number of Twitter followers an individual user has can be operationalized as indegree centrality. Here, indegree centrality reflects the extent to which a user is the object of relationships, operationalized as 'followers.' We expect that higher in-degree centrality is positively related to the extent to which messages are retweeted. Therefore, we propose the following:

H4: Message source indegree centrality is positively related to retweeting frequency.

Engagement in social media has been assessed in a variety of ways. Cha et al. [9] calculated influence with in-degree, out-degree, mentions, and retweeting behavior. This evidence suggests that messages created by Twitter users who are higher in prestige or status should exhibit higher levels of diffusion [9]. Building on this research, Stefanone, Kwon, and Lackaff [44] developed and tested a measure of social prestige to assess the relative impact social media can have on outcomes like social capital. In their work, social status was operationalized as participant indegree - outdegree where outdegree is measured as the number of individuals a particular user follows. In other words, if a Twitter user has 100 followers, and follows only 20 others, their status level is +80 . On the other hand, if they have 20 followers and follow 100 others, their status level equals -80 . We hypothesize that messages from users high in social status are retweeted more often. Thus,

H5: The social status of message sources is positively related to retweeting frequency.

\subsection{Message-level controls}

Accounting for audience characteristics alone may be inadequate, and additional message-level and temporal controls may further clarify the impact of the uniqueness of different users has on social media use.

One of the most interesting innovations in social media messages is the hashtag. Hashtags are generally considered as "topics" or issue involvement [10], and have been found to be strongly associated with retweetability in early Twitter research [48]. The causal mechanism plausibly lies in how a hashtag connects the message to what Bruns and Burgess [7] call the "ad hoc public" that coalesces around the hashtag-based discussions. We posit that tapping into existing publics through the use of hashtags will be associated with greater diffusion; thus,
H6: Hashtag use is positively related to retweeting frequency.

Finally, public sphere issues discussed via social media are event-driven and dynamic. In the context of news coverage, the amount of attention given to issues in online discussions is often driven by traditional mass media coverage of major events, consistent with agenda setting principles of mass media. Images shared via Twitter sent immediately following major events should be more likely to diffuse because they have high salience and are most relevant at that moment. Additionally, Downs [14] noted that attention to topics is related to the timeliness of salient events. As such, as time passes after events that garner national news coverage, images will be less likely to propagate across social media. Thus, the following hypothesis is proposed:

H7: Time after news coverage of national events has a negative relationship with retweeting frequency.

\section{Method}

We use the case of the gun control-related conversations mediated by Twitter to examine how image attributes like appeal, frame and valence predict diffusion. Throughout, we operationalize diffusion as retweeting frequency of messages with images related to gun control.

Gun control is a controversial issue in the United States and Americans are politically divided on this issue. For example, a CBS/NYTimes poll of 1,644 adults conducted in February 2014 shows that 54\% of Americans think that gun control laws should be more strict.

Various parties use social media to promote pro and anti-gun sentiment online. Nearly 21 million Twitter messages on gun control were sent during the four-month period after Newton school shooting in Connecticut in late 2012 [30]. Exploratory analysis into gun control tweets also found that the \#guncontrol tag was used by both pro- and anti-gun control supporters. The timeliness of gun control as well as its popularity on Twitter provide an active and appropriate communication forum to test the hypotheses presented herein. What follows is a description of the methods used for data collection followed by the presentation and discussion of results.

Additionally, all major spree shootings in the U. S. were tracked from September 1st 2013 through October 15th 2013. There were three shooting events that gained national attention during this time frame: 
the Washington Navy Yard on September 16 (8 wounded, 13 killed), Pine Hills High School on October 4 (2 wounded), and Lanier High School on October15 (1 wounded).

\subsection{Data Collection}

Python (v.3) was used to create a Twitter crawler to collect all tweets containing the hashtag guncontrol (\#guncontrol) from the 1st through 15th of October 2013. This time frame was selected due to its proximity to recent shooting events (discussed below). A total of 15,840 tweets were collected during this time. 8,306 were original tweets (opposed to RTs). After limiting the dataset to original tweets, we selected all tweets with a media_url variablethis indicates if the tweet contains an imagereducing the sample to 486 tweets that were both original and included an image.

Analyses were limited to the use of still images. These included photographs, photographs with text overlay, cartoons, and charts. GIFs, videos, and dynamic content were excluded. Many tweets were unique insofar as the Twitter user posted content to their network that was not a RT, but drew on the same source for both image and text overlay (if applicable). In this way, duplicates emerged in original media messages. Filtering out those duplicates reduced the sample to 290 images.

3.1.1. Content Analysis. Content analysis is a research technique that is objective, systematic, and often quantitative in nature [5]. Coders were instructed to focus on the primary element of each coded category. Categories were selected based on previously reviewed literature (see Appendix 1 for codebook, definitions, and frequencies) and were independently cross-coded by two trained coders. Coders trained independently over three sessions of 30 images each, meeting after each session to refine technique. Each session lasted approximately 20-30 minutes to review and refine technique for the sample set.

Additionally, the entire sample of 290 images was cross-coded by both coders to insure reliability throughout. During the final round of coding, all disagreements were resolved between coders creating a complete and mutually agreed upon corpus of images and codes. All coding schemes were based on identification of a primary category; even if multiples appeals or frames exist, only the most dominant scheme was used for coding purposes.

The nine individual appeal types shown in the Appendix plus a tenth category for 'no/other appeal' formed the first block of the coding scheme yielding high Cohen's Kappas $(\kappa=.955)$. Three types of frames - risk, attribute, and goal-formed the second block $(\kappa=.933)$; messages with no apparent frame were given a 'no frame/other' code. Valence, in turn, was coded as the 'intended valence' (neutral, negative, or positive) the coder believed the message creator was trying to evoke $(\kappa=.897)$.

In a final step, dummy variables were created out of each of the above codes to facilitate testing in a series of multivariate regressions.

Each category was assessed independently with a Cohen's Kappa ( $\kappa)$. All categories scored well above .85 , and any of the disagreements noted were resolved prior to analysis, resulting in $100 \%$ agreement between coders.

\section{Results}

The images coded fell into two broad appeal classes of emotion based and thought solicitations. Nearly half of the images focused on emotional solicitations (48.7\% included humor, fear, sex, or broad emotion appeals) while a third of images focused on thought based solicitations $(32.5 \%$ one or two sided messages, metaphor, rational, or ethos appeals) with remaining images either lacked an appeal or were unclear in their intention.

Just over a third of all images contained identifiable frames $(38.8 \%)$ with the vast majority focusing on either attribute $(12.1 \%)$ or goal framing $(22.4 \%)$ as their primary focus. Valence was similarly distributed with neutral (50.3\%) and negative $(40.0 \%)$ representing the majority of the sample, with the remaining portion allocated to positively valenced images $(9.7 \%)$.

The users were diverse in their attributes, with accounts ranging from 0 to 54,417 followers $(\mathrm{M}=$ $2,388,11, S D=5,036.85)$ and hashtag usage varying from 1 to 15 tags per tweet $(\mathrm{M}=3.71, S D=2.60)$. Temporally, the time between shooting incidents was on average one week $(\mathrm{M}=6.94, S D=5.71)$. Collectively, the tweets received between 0 and 71 RT $(\mathrm{M}=1.35, S D=4.04)$. In the analyses presented the omitted groups (no/other appeals, neutral valence, no/other frame) served as the comparison point for the others. In presenting data in this fashion, it is evident how the inclusion of a variable or category of a variable directly impacts outcomes (in this case, retweeting behavior).

The data overall represented significant diversity both in message construction and user competence in cultivating twitter presence in the form of high followership. This manifested in significant variation in RT behavior as individuals attempted to share information with varying degrees of success through 
Table 1. Descriptive statistics.

\begin{tabular}{|c|c|c|c|c|}
\hline Category & $\begin{array}{l}\text { Sub- } \\
\text { Category }\end{array}$ & $\mathrm{N}$ & Percent & $\begin{array}{l}(\text { mean }) \\
\min / \max \end{array}$ \\
\hline \multirow[t]{13}{*}{ Appeal } & & 290 & 100 & \\
\hline & Fear & 37 & 12.8 & \\
\hline & Humor & 52 & 17.9 & \\
\hline & One or Two & 13 & 4.5 & \\
\hline & Sided & & & \\
\hline & Message & & & \\
\hline & Sex & 6 & 2.1 & \\
\hline & Metaphor & 15 & 5.2 & \\
\hline & Threat & 0 & 0 & \\
\hline & Emotional & 46 & 15.9 & \\
\hline & Rational & 73 & 25.2 & \\
\hline & Ethos & 8 & 2.8 & \\
\hline & $\begin{array}{l}\text { No Appeal } \\
\text { /Other }\end{array}$ & 40 & 13.8 & \\
\hline \multirow[t]{5}{*}{ Frame } & & 290 & 100 & \\
\hline & Attribute & 35 & 12.1 & \\
\hline & Valence & 2 & 0.7 & \\
\hline & Goal & 65 & 22.4 & \\
\hline & $\begin{array}{l}\text { No } \\
\text { frame/Other }\end{array}$ & 188 & 64.8 & \\
\hline \multirow[t]{4}{*}{ Valence } & & 290 & 100 & \\
\hline & Positive & 28 & 9.7 & \\
\hline & Neutral & 146 & 50.3 & \\
\hline & Negative & 116 & 40.0 & \\
\hline \multirow{8}{*}{$\begin{array}{l}\text { User and } \\
\text { Tweet } \\
\text { Controls }\end{array}$} & & 486 & & \\
\hline & & & & \\
\hline & Number of & & & $(2,388.11)$ \\
\hline & Followers & & & $0 / 54,417$ \\
\hline & $\begin{array}{l}\text { Number of } \\
\text { Hashtags }\end{array}$ & & & \\
\hline & Time Since & 3 & & \\
\hline & Last Event & & & (6.94) \\
\hline & & & & $0 / 17$ \\
\hline \multirow{3}{*}{$\begin{array}{l}\text { Number } \\
\text { of RT }\end{array}$} & & 486 & & (3.71) $1 / 15$ \\
\hline & Retweet & & & \\
\hline & Frequency & & & (1.35) $0 / 71$ \\
\hline
\end{tabular}

their respective networks. Findings indicate that users who are successful in having messages RT need more than large networks of people with access to their content (see: Table 2). The hypotheses presented herein focused on the diffusion and processing of different tweet content across appeal, frame, valence, and user-level, message-level, and temporal characteristics. A series of three negative binomial regressions (see table 2) were conducted to test the hypotheses, one each for the frame, valence, and appeal measures (sender and message controls are included in all models). The negative binomial model is designed for ratio-level count-dependent variables
Table 2. Negative Binomial Regression predicting retweet frequency.

Frame $\quad$ Valence $\quad$ Appeal

$\begin{array}{lc}\text { Appeal: } & \mathbf{0 . 8 8} * \\ \text { Fear } & \mathbf{( 0 . 4 4 )} \\ & \mathbf{0 . 6 9} \\ \text { Humor } & \mathbf{( 0 . 4 1 )} \\ & -0.02 \\ 1 \text { and } 2 & (0.61) \\ \text { Sided } & 0.31 \\ \text { Sex } & (0.76) \\ & 0.72 \\ \text { Metaphor } & (0.54) \\ \text { Emotional } & 0.48 \\ & (0.42) \\ \text { Rational } & 0.44 \\ \text { Ethos } & (0.39) \\ & 0.44 \\ & (0.75)\end{array}$

$\begin{array}{lc}\text { Frame: } & \\ \text { Attribute } & \mathbf{0 . 5 9} \boldsymbol{\ell} \\ & \mathbf{( 0 . 3 2 )} \\ \text { Valence } & 0.70 \\ & (1.36) \\ \text { Goal } & -0.16 \\ & (0.27)\end{array}$

Valence:

Positive $\quad 0.81$ *

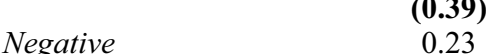

$(0.24)$

Controls:

$\begin{array}{lccc}\text { Number of } & \mathbf{0 . 3 5 * *} & \mathbf{0 . 3 2} * * & \mathbf{0 . 3 5 * *} \\ \text { followers } & \mathbf{( 0 . 0 6 )} & \mathbf{( 0 . 0 6 )} & \mathbf{( 0 . 0 6 )} \\ \text { Number of } & 0.07 & \mathbf{0 . 0 8 *} & \mathbf{0 . 0 7} \text { : } \\ \text { Hashtags } & (0.04) & \mathbf{( 0 . 0 4 )} & \mathbf{( 0 . 0 4 )} \\ \text { Time Since } & \mathbf{- 0 . 0 4 *} & \mathbf{- 0 . 0 4 *} & \mathbf{- 0 . 0 5 * *} \\ \text { Last Event } & \mathbf{( 0 . 0 2 )} & \mathbf{( 0 . 0 2 )} & \mathbf{( 0 . 0 2 )} \\ N & 290 & 290 & 290 \\ \text { Pseudo } \mathrm{R}^{2} & 0.159 & 0.156 & 0.155 \\ \text { Model Sig. } & 50.26^{* *} & 49.29 * * & 48.69^{* *} \\ \left.\chi^{2}\right) & & & \\ \text { Log } & -377.14 & -377.63 & -377.92\end{array}$

Likelihood

Note* Standard Errors in parentheses; $\boldsymbol{\ell} p<.10, * p<.05$, ** $p<.01$. With maximum-likelihood models such as negative binomial regression, there is no traditional $\mathrm{R}^{2}$; for this reason, an analogous 'pseudo- $\mathrm{R}^{2}$ ' is reported; The $\mathrm{R}^{2}$ shown here is the ML (Cox-Snell) $\mathrm{R}^{2}$.

such as those collected in this study. To deal with such data, researchers typically employ various nonlinear models based on the Poisson and negative binomial distributions. In this case, the dispersion for 
the count variable (number of retweets) is greater than would be expected for a traditional Poisson distribution (the variance is much larger than the mean); thus, the models are estimated using the negative binomial technique.

The model in table 2 evaluating appeal types was significant, $\chi^{2}=48.69, \mathrm{p}<.01$, and had a Cox-Snell $\mathrm{R}^{2}$ of 0.16 . The results show that messages with images that have both fear and humor appeals were more likely to be retweeted than either the baseline (omitted) category of "no/other appeal" or the other five appeal types. Thus H1a and $1 \mathrm{~b}$ were supported. With regard to frame type, again the model was significant, $\chi^{2}=50.26, \mathrm{p}<.01$. Images with attribute framing were more likely to be retweeted than the baseline category of 'no frame' as well valence or goal frames, but this finding was significant at only the $\mathrm{p}<.10$ level. Thus $\mathrm{H} 2$ was weakly supported. Next, the model for valence was significant, $\chi^{2}=$ $49.29, \mathrm{p}<.01$. The significant, positive coefficient on "Positive" indicates that positively valenced messages are associated with significantly greater retweets than tweets with a neutral valence (the baseline category), supporting $\mathrm{H} 3$.

Hypotheses 4 through 7 address how user-level and context characteristics impact retweeting behavior. The number of followers was a significant predictor in each model. More followers positively related to retweeting behavior, support for $\mathrm{H} 4$. Surprisingly, social status (or, prestige) was not significant in any of the models. It was excluded from table 2, as H5 was not supported. Results also show that hashtags were a significant predictor of retweets $(p<.05)$ in two of the three models. Thus, H6 was largely supported. Finally, H7 focused on the timeliness of shooting events and the relationship to diffusion. As predicted, the time since shooting events had a negative relationship with retweeting behavior, such that the longer it had been since an incident the fewer times images were shared.

\section{Discussion}

We live in a rapidly evolving mass media context where information is created through decentralized peer-to-peer processes and shared via social media. Increasingly, these social media messages - especially those that go viral - assume a multimodal or purely graphical form. While scholars have recognized the potential for diffusion of imagebased messages, they have yet to study in detail which characteristics of the images are systematically related to diffusion online. This study helps address this gap in the literature. This is, to our knowledge, the first study to examine the relationship between specific image characteristics and how widely messages shared via social media propagate across Twitter networks.

To help explain message diffusion rates we develop a series of hypotheses that tap a diverse array of message, audience, tagging, and temporal characteristics. Our findings carry several implications for future research on the diffusion of messages (and images) via social media.

To start, we theorized for variable diffusion effects according to the type of appeal and the message's valence and framing. Our study is perhaps the first to incorporate a focus on the psychological orientation of social media messages. The findings support our hypotheses regarding the types of appeals that make a message "shareable." Specifically, fear and humor appeals are more likely to get messages broadly disseminated while, in contrast, neither emotional nor rational appeals exhibited a significant relationship with diffusion. Interestingly, while sex may "sell," in this context sex appeals were not significantly related to retweetability.

We also found that messages with attribute frames and positive valences were more likely to be retweeted. Our study thus adds to the debate about the diffusion of positive vs. negative messages [6, 24], although is limited in its ability to explain why positively valenced messages are more likely to diffuse. Perhaps positively valenced messages were more popular because they functioned as a balance to the predominantly negative content characterizing mass media coverage of gun violence.

Perhaps surprisingly, results show that our specific measure for social status of message sources did not predict diffusion. Recall that status was operationalized as indegree - outdegree. Traditionally message source characteristics like status or prestige do influence the attention audiences give to messages, and subsequent message influence. It may be that decentralized and large scale online networks like Twitter are deemphasizing the importance of message sources. This calls into question many issues related to information accuracy and validity in terms of news content, and outcomes related to message framing and public understanding of complex issues in the public sphere. This is certainly an area for further research.

Our study also integrated a series of user, tweet, and temporal characteristics. We found each of these variables - the number of followers, the number of hashtags, and the time since the last mass shooting event - were significantly related to tweeting behavior. In particular, we found that the most highly retweeted messages were those sent by users with a 
greater number of followers, that connected to more existing issues through the use of hashtags, and occurred more closely in time to a mass shooting event. Perhaps audience size alone is a more effective measure of user status.

These findings thus conform to other research on the size of the audience in other contexts (e.g., [38]) while providing further support to the relevance of hashtags and pre-existing discussions in a range of social media-based communicative phenomena. To our knowledge, we may be also the first to explicitly incorporate the temporal dimension into an analysis of the diffusion of social media messages.

At the broadest level, our results suggest that analyses of message diffusion via social media systems should strive to be multi-level and multidimensional in nature. We have found that diffusion is related to not only the content (hashtags) and psychological orientation (frame, valence, and appeal type) of the message but also the size of the audience - a key attribute of the sender - along with the temporal context in which the message is sent. It is our hope that future research will recognize the richness of the determinants of message diffusion and refrain from testing models at only the user or tweet level or that ignore the dynamic, temporally dependent nature of message diffusion on a real-time, inter-connected message network such as Twitter.

Of course, our study was conducted in the context of one specific issue (gun control) during a limited time period (fall 2013); generalizations to other contexts should therefore be made cautiously. However, given the theoretical basis of the hypotheses presented, we believe that these findings should generalize to other kinds of messages (e.g., text).

Although our role in the media ecosystem has changed from consumer to producer, and social media facilitates the creation and dissemination of a wide range of content, many social media platforms have been appropriated for the dissemination of news-related information in particular. The selective media experience raises many more questions about the relationship between content characteristics and diffusion beyond the variables addressed in this study. As individuals become more discerning about the media they attend to, they rely on an increasingly diverse and decentralized pool of information sources. This has obvious implications for the diffusion of and access to news-related content.

We believe these findings carry valid and notable implications for scholars wishing to understand what leads users to share messages in this rapidly evolving mass media context of the social media era.

\section{References}

[1] I. Anger, and C. Kittl, Measuring influence on Twitter", In I-KNOW 31, September, 2011.

[2] E. Bakshy, J. M. Hofman, W. A. Mason, and D. J. Watts, "Everyone's an influencer: Quantifying influence on twitter", Proceedings of the 4th ACM International conference on Web search and Data Mining, WSDM, 2011, pp. 65-74.

[3] E. Bakshy, I. Rosenn, C. Marlow, and L, Adamic, "The role of social networks in information diffusion", In Proceedings of the 21st international conference on World Wide Web, ACM, April, 2012, pp. 519-528.

[4] S. Bennett, "IT Marketing and the Rise of Social Media", Mediabistro.com, 1/29/14.

[5] Berelson, B. Content analysis in communication research. Free Press, New York, 1952.

[6] B. Y. Bizer, J. T. Larsen, and R. E. Petty, "Exploring the valence-framing effect: negative framing enhances attitude strength", Political Psychology, 3, 2010, pp. 1-22.

[7] A. Bruns, and J. E. Burgess, "The use of Twitter hashtags in the formation of ad hoc publics", In 6th European Consortium for Political Research General Conference, University of Iceland, Reykjavik August, 2011, pp. 25 - 27.

[8] J. J. Burnett, and R. L. Oliver, "Fear appeal effects in the field: A segmentation approach", Journal of Marketing Research, 16,1979, pp. 181-90.

[9] M. Cha, H. Haddadi, F. Benevenuto, and P. K. Gummadi, "Measuring User Influence in Twitter: The Million Follower Fallacy”, ICWSM 10,2010 , pp. 10-17.

[10] J. Chen, and P. Pirolli, "Why you are more engaged: factors influencing twitter engagement in occupy Wall Street", Proceedings of the Sixth International AAAI Conference on Weblogs and Social Media, 2012.

[11] M. D. Choudhury, Y. R. Lin, H. Sundaram, K. S. Candan, L. Xie, and A. Kelliher, "How does the data sampling strategy impact the discovery of information diffusion in social media?", Proceedings of the fourth international AAAI conference on weblogs and social media, 2010, pp. 34-41.

[12] Cialdini, R. B. Influence: Science and Practice, (4th Ed), Allyn and Bacon, Boston, 2001.

[13] C. J. Chung, Y. Nam, and M. A. Stefanone, "Exploring Online News Credibility: The Relative Influence of Hypertextuality, Interactivity and Multimediality", Journal of Computer-Mediated Communication, 17, 2012, pp. 171-186.

[14] A. Downs, "Up and down with ecology: The issue attention cycle", Public interest, 28, 1972, pp. 38-50.

[15] R. M. Entman, "Framing: Towards clarification of a fractured paradigm", Journal of Communication, 43, 1993, pp. 5158.

[16] M. E. Grabe, and E. P. Bucy, "Image bite politics: news and the visual framing of elections", Public Opinion Quarterly, 73 , 2009, pp. 595-598. 
[17] L. K. Hansen, A. Arvidsson, F. Å. Nielsen, E. Colleoni, and M. Etter, "Good friends, bad news-affect and virality in twitter", In Future information technology, Berlin Heidelberg. Springer, 2011.

[18] I. Himelboim, S. McCreery, and M. Smith, "Birds of a Feather Tweet Together: Integrating Network and Content Analyses to Examine Cross-Ideology Exposure on Twitter", Journal of Computer-Mediated Communication, 18, 2013, pp. 40-60.

[19] L. Hong, O. Dan, and B.D. Davison, "Predicting popular messages in twitter", In Proceedings of the 20th international conference companion on World wide web, ACM, March, 2011, pp. 57-58.

[20] C. R. Hullett, "The impact of mood on persuasion. A meta-analysis", Communication Research, 32, 2005, pp. 423-442.

[21] D. Kahneman, and A. Tversky, "Choices, Values, and Frames”, American Psychologist, 39, 1984, pp. 341-350.

[22] P. Krishnamurthy, P. Carter, and E. Blair, "Attribute framing and goal framing effects in health decisions", Organizational Behavior and Human Decision Processes, 85, 2001, pp. 382-399.

[23] Kuhberger, A, Encyclopiedia of Life Support Systems, Framing Effects in Theory and in Practice. Optimization and Operations Research, 6, Eolss Publishers, Oxford, UK, 1998

[24] J. Lin, and J. Pena "Are you following me? A content analysis of tv networks' brand communication on twitter"; Journal of Interactive Advertising, 12, 2011, pp. 17-29.

[25] S. Lio, S. Melzer, and E. Reese, "Constructing threat and appropriating "Civil Rights": Rhetorical strategies of gun rights and English only leaders", Symbolic Interaction, 31, 2008, pp. 5-31.

[26] Lowery, S. A. and M. L. DeFleur, Milestones in Mass Communication Research. Longman Publishers. White Plains, NY, 1995.

[27] N. R. Maier, "Assets and liabilities in group problem solving: The need for an integrative function", Psychological Review, 74,1967, pp. 239-249.

[28] P. M. Napoli, and N. Gillis, "Reassessing the potential contribution of communications research to communications policy: The case of media ownership", Journal of Broadcasting and Electronic Media, 50, 2006, pp. 671-691.

[29] J. Panksepp, and J. Burgdorf, "Laughing rats? Playful tickling arouses high frequency ultrasonic chirping in young rodents", Toward a science of Consciousness III, MIT Press, Cambridge, 1999, pp. 231-244.

[30] Pew Research Center's Journalism Project Staff. "Gun Control and the Media", April 25, 2013. Accessed: http://www.journalism.org/2013/04/25/spikes-attention-and-shiftsopinion-gun-control-debate/

[31] J. R. Priester, and R. E. Petty, "The influence of spokesperson trustworthiness on message elaboration, attitude strength, and advertising effectiveness", Journal of Consumer Psychology, 13, 2003, pp. 408-421.
[32] Ramachandran, V.S. and S. Blakeslee, Phantoms in the Brain. William Morrow, N.Y., 1998.

[33] J. Ratkiewicz, M. Conover, M. Meiss, B. Goncalves, S. Patil, A. Flammini, and F. Menczer, "Truthy: mapping the spread of astroturf in microblog streams", Proceedings of the 20th international conference companion on world wide web, 2011, pp. 249-252.

[34] V. J. Rideout, U. G. Foehr, D. F. Roberts, "Media in the lives of 8-18 year-olds", Generation $\mathrm{M}^{2}$, Kaiser Family Foundation, 2010.

[35] Rogers, E. M. Diffusion of innovations (5th edition), Free Press,New York, NY.

[36] A. J. Rothman, S. C. Martino, B. T. Bedell, J. B. Detweiler, and P. Salovey, "The systematic influence of gain- and loss-framed messages on interest in and use of different types of health behavior", Personality and Social Psychology Bulletin, 25, 1999, pp. 1355-1369. ]

[37] J. Rui, and M. A. Stefanone, "Strategic self-presentation online: A cross-cultural study", Computers in Human Behavior, 29, 2013, pp. 110-118.

[38] G. D. Saxton, and R. D. Waters, "What do stakeholders like on Facebook? Examining public reactions to nonprofit organizations' informational, promotional, and communitybuilding messages", Journal of Public Relations Research. Advance online publication. Forthcoming.

[39] D. A. Scheufele, and D. Tewksbury, "Framing, Agenda Setting, and Priming: The Evolution of Three Media Effects Models", Journal of Communication, 57, 2007, pp. 9-20.

[40] Schramm, W. and D. F. Roberts, The process and effects of mass communication. University of Illinois Press.1971.

[41] H. Shosteck, R. F. Clarke, "Need for information on adverse drug reactions including the reactions of present and potential readers to clinical experience abstracts", US National Technical Information Service, 1973, pp. 234-724.

[42] K. Starbird, G. Muzny, and L, Palen, "Learning from the crowd: Collaborative filtering techniques for identifying on-theground twitterers during mass disruptions", Proceedings of the 9th international ISCRAM conference, 2012, pp. 1-10.

[43] M. A. Stefanone, C. M. Hurley, and J. Z. Yang, "Antecedents of online information sharing", Information, Communication, and Society, 16, 2013, pp. 61-81.

[44] M. A. Stefanone, K. H. Kwon, and D. Lackaff, "The relationship between perceptions of online capital and enacted support", Journal of Computer-Mediated Communication, 17, 2012, pp. 451-466.

[45] M. A. Stefanone, D. Lackaff, and D. Rosen, "The Relationship between traditional mass media and "social media": Reality television as a model for social network site behavior", Journal of Broadcasting and Electronic Media, 54, 2010, pp. 508525.

[46] S. Stieglitz, and L. Dang-Xuan, "Political Communication and Influence through Microblogging - An Empirical Analysis of Sentiment in Twitter Messages and Retweet Behavior", In Proceedings of the 45th Hawaii International 
Conference on System Sciences (HICSS), Grand Wailea, Maui, Hawaii, 2012, pp. 3500-3509.

[47] D. Strang, and S. A. Soule "Diffusion in organizations and social movements: from hybrid corn to poison pills", Annual Review of Sociology, 24, 1998, pp. 265-290.

[48] B. Suh, L. Hong, P. Pirolli, and E. H. Chi, "Want to be retweeted? Large scale analytics on factors impacting retweet in twitter network", IEEE 2010 Second International Conference on Social Computing, 2010, pp. 177-184.

[49] V. C. Troldahl, "A Field Test of a Modified Two-Step Flow of Communication Model", Public Opinion Quarterly, 30, 2010, pp. 609-623.

[50] D. Westerman, P. R. Spence, and B. Van Der Heide, “A social network as information: The effect of system generated reports of connectedness on credibility on Twitter", Computers in Human Behavior, 28, 2012, pp. 199-206.

[51] K. Witte, and M. Allen, "A meta-analysis of fear appeals: Implications for effective public health campaigns", Health Education Behavior, 27, 2000, pp. 591-615.

[52] W.W. Xu, Y.M. Sang, S. Blasiola, and H.W. Park, "Predicting opinion leaders in Twitter activism networks: The case of the Wisconsin recall election", American Behavioral Scientist. Forthcoming.

[53] A. Mitchell, M. Jurkowitz, "The Gun Control Debate," Pew Research Center.

\section{Appendix}

\begin{tabular}{|c|c|}
\hline Category Type & Definition \\
\hline \multicolumn{2}{|l|}{ Appeal } \\
\hline Fear & $\begin{array}{l}\text { Message that evokes negatively } \\
\text { valence emotion with a high level } \\
\text { of arousal. Causes a sense of } \\
\text { dread / foreboding. Apprehension } \\
\text { of threat, danger or pain. }\end{array}$ \\
\hline One/two sided & $\begin{array}{l}\text { Message that shows either one or } \\
\text { multiple facets of an argument. } \\
\text { Can compare and contrast } \\
\text { information - information does } \\
\text { not need to be truthful. }\end{array}$ \\
\hline Sex & $\begin{array}{l}\text { Message intended to entice via } \\
\text { sexual or physically attractive } \\
\text { traits. Can include sultry dress, } \\
\text { imply intercourse, or sexually } \\
\text { charged situations. }\end{array}$ \\
\hline Metaphor & $\begin{array}{l}\text { Message that contains an implied } \\
\text { comparison between two } \\
\text { dissimilar objects. }\end{array}$ \\
\hline Threat & $\begin{array}{l}\text { Message that evokes negatively } \\
\text { valence emotion accompanied by } \\
\text { a threat that is severe but also } \\
\text { presents a workable solution. The } \\
\text { solution differentiates threat from } \\
\text { fear. }\end{array}$ \\
\hline Emotional & $\begin{array}{l}\text { Message that elicits emotion other } \\
\text { than fear. }\end{array}$ \\
\hline Rational & $\begin{array}{l}\text { Message that contains facts or } \\
\text { pseudo-facts intended to } \\
\text { persuade. Can be a table of data, } \\
\text { or statistics. }\end{array}$ \\
\hline
\end{tabular}

\begin{tabular}{|l|l|}
\hline Ethos & $\begin{array}{l}\text { Message that appeals to a person's } \\
\text { character or morality. }\end{array}$ \\
\hline Humor & $\begin{array}{l}\text { Message that evokes laughter, } \\
\text { often through incongruence or the } \\
\text { combination of unexpected } \\
\text { things. Amusing or comical. }\end{array}$ \\
\hline No/other & $\begin{array}{l}\text { Message that does not clearly fit } \\
\text { into the above categories. }\end{array}$ \\
\hline Frames & $\begin{array}{l}\text { Defined as risky frame } \\
\text { presentation where choices are } \\
\text { presented positively or } \\
\text { negatively. }\end{array}$ \\
\hline Valence & $\begin{array}{l}\text { Defined as emphasizing a single } \\
\text { facet positively or negatively. }\end{array}$ \\
\hline Attribute & $\begin{array}{l}\text { Defined as emphasizing outcomes } \\
\text { as either positive or negative. }\end{array}$ \\
\hline Goal & No obvious frame present. \\
\hline No frame & $\begin{array}{l}\text { A negative emotion (sadness, } \\
\text { anger, contempt, disgust, fear) } \\
\text { appeared to be intended by the } \\
\text { sender. }\end{array}$ \\
\hline Intended Valence & $\begin{array}{l}\text { No obvious emotional state, } \\
\text { positive or negative, appeared to } \\
\text { be intended by the sender } \\
\text { (includes surprise). }\end{array}$ \\
\hline Negative valence \\
\hline Positive valence & $\begin{array}{l}\text { A positive emotion (happiness) } \\
\text { appeared to be intended by the } \\
\text { sender. }\end{array}$ \\
\hline
\end{tabular}

\section{Note: This research was funded by AFOSR grant FA9550-12-1-0201.}

